\title{
Radio, televisión, audio y vídeo en educación. Funciones y posibilidades, potenciadas por el COVID-19
}

\section{(Radio, Television, Audio and Video in Education. Functions and Possibilities, Enhanced by COVID-19)}

\author{
Lorenzo García Aretio \\ UNED (España)
}

DOI: https://doi.org/10.5944/ried.25.1.31468

\section{Cómo referenciar este artículo:}

García Aretio, L. (2022). Radio, televisión, audio y vídeo en educación. Funciones y posibilidades, potenciadas por el COVID-19. RIED. Revista Iberoamericana de Educación a Distancia, 25(1), pp. 9-28. https://doi.org/10.5944/ried.25.1.31468

\section{Resumen}

El medio audiovisual está tan extendido que fue apuesta educativa en entornos a distancia y ahora, de forma notable, durante los tiempos de pandemia. Esos recursos audiovisuales bien utilizados potencian las posibilidades de aprendizaje y retención. A pesar de que hoy muchos podrían pensar que se trata de recursos obsoletos, de escasa eficacia para el aprendizaje en esta sociedad digital, siguen siendo hoy bien valorados. Fue evidente que la radio y la televisión tuvieron una destacada relevancia en los tiempos de confinamiento, con el propósito de que nadie se quedase atrás, sobre todo, los grupos más vulnerables. Hoy, los sistemas digitales pueden alojar, grabar y reproducir cualquier tipo de documento audiovisual, y esto último, sea en formato síncrono o asíncrono. La radio, en tiempos de pandemia se aprovechó como medio de comunicación flexible, masivo, de bajo coste y de largo alcance, como alternativa a los grandes avances de las tecnologías digitales, llegando a todos los rincones del globo. Sobre la radio, el podcast cuenta con la ventaja de poder grabar y reproducir cualquier tipo de contenido sonoro, con posibilidades de escucha reiterada, pausa y vuelta atrás. Por una parte, integrar oído y vista, por la otra, el texto, audio e imágenes en un mismo recurso educativo, casos de la televisión y el vídeo, potencian de forma sustancial las posibilidades didácticas en cualquier área del saber, índole del curso y nivel educativo. Sin embargo, ha de concluirse que el potencial de estos recursos no se ha explotado, ni mucho menos, lo suficiente en entornos educativos. La videoconferencia síncrona parece haber integrado las posibilidades de estas herramientas, obviando algunos de sus problemas y convirtiéndose en gran complemento para la educación. 
Palabras clave: audio; radio; vídeo; televisión; comunicación audiovisual; COVID-19.

\begin{abstract}
The audiovisual medium is so widespread that it was an educational bet in remote settings and now, notably, during the times of the pandemic. These well-used audiovisual resources enhance the possibilities of learning and retention. Despite the fact that, nowadays, many might think that these are obsolete resources, of low efficiency for learning in this digital society, they are still highly valued today. It was evident that radio and television had an outstanding relevance in times of confinement, so that no one was left behind, especially the most vulnerable groups. Today, digital systems can host, record and reproduce any type of audiovisual document, and the latter, both in synchronous and asynchronous format. Radio, in times of pandemic, was used as a flexible, massive, low-cost and long-range means of communication, as an alternative to the great advances in digital technologies, reaching all corners of the globe. Over the radio, the podcast has the advantage of being able to record and reproduce any type of audio content, with the possibility of repeated listening, pausing and going back. On the one hand, integrating hearing and sight, on the other hand, text, audio and images in the same educational resource, such as television and video, substantially enhance the didactic possibilities in any area of knowledge, nature of the course and educational level. However, it must be concluded that the potential of these resources has not been exploited, far from it, sufficiently in educational settings. Synchronous videoconferencing seems to have integrated the possibilities of these tools obviating some of their problems and becoming a great complement to education.
\end{abstract}

Keywords: audio; radio; video; television; audiovisual communication; COVID-19.

Nuestros hogares cuentan, y contaron desde décadas atrás, con medios audiovisuales de uso ordinario (radio, TV, vinilo, casete, vídeo, CD, DVD, Blu-ray, etc.). El medio audiovisual está tan extendido que fue la gran apuesta educativa de muchos años (Bartolomé, 2000), especialmente en formatos no presenciales y, ahora de forma notable, durante los tiempos de pandemia. En el presente trabajo se pretenden ofrecer razones sobre el servicio educativo que pueden brindar estos recursos, sobre todo en los sistemas educativos a distancia y digitales, para reforzar la presencia social del docente y para hacer más eficaces los procesos de realimentación o feedback. Esos recursos audiovisuales, bien utilizados, potencian la capacidad de aprendizaje y retención, pueden suplir tareas rutinarias y mecanicistas del docente, así como presentar hechos y fenómenos de difícil contacto o visión directa (García Aretio, 2001; Cabero y Gisbert, 2005).

Cierto que la televisión y la radio educativas, en aquellos inicios prometedores, se tropezaron con algunas realidades que frenaron las ilusiones iniciales. El problema de los horarios condicionaba mucho el seguimiento de determinados programas por parte del alumnado, porque la relación debía ser síncrona para poder beneficiarse del programa en cuestión. Además, el carácter de comunicación, básicamente 
unidireccional de estos medios, limitaba en cierto modo sus posibilidades educativas (Baum y McPherson, 2019). A pesar de esos obstáculos reales y de que hoy muchos podrían pensar que se trata de recursos obsoletos, de escasa eficacia en el aprendizaje en esta sociedad digital, medios como la radio y la televisión son actualmente bien valorados (Rodas y Celleri, 2017; Grupo de Trabajo sobre Tecnología e Innovación en la Educación, 2020).

Más allá de estas consideraciones genéricas sobre el valor del medio audiovisual, en marzo de 2020 llegó la pandemia y el subsiguiente cierre total de instalaciones educativas y los posteriores cierres parciales o intermitentes. Esas circunstancias fueron las que invitaron a proponer que el presente monográfico de RIED se centrase en esas potentes herramientas de comunicación electrónica o digital, a través del audio y el vídeo. Porque fue evidente que recursos como la radio o la televisión tuvieron una destacada relevancia en los tiempos de confinamiento, con el propósito de que nadie se quedase atrás, sobre todo, los grupos más vulnerables que podrían quedar más lejos de determinadas dotaciones de conectividad o dispositivos digitales. Estos medios cobraron un gran sentido, dado que en muchas situaciones no se disponía de otros recursos para hacer llegar la educación (Makazaga, 2020) o cuando, simplemente, el aprendizaje en línea era imposible o de difícil acceso (UNESCO, 2020).

Datareportal (2021) informa que, en este año 2021, algo más del 60\% de la humanidad tiene acceso a Internet, pero, leyéndolo de otra forma, quiere decirse que algo menos del $40 \%$ de esa población mundial no cuenta con conectividad a la red. Más allá de que muchas de las personas que conforman ese $60 \%$ de privilegiados no disfruta de una conexión fiable, consistente y estable (Figura 1) como para garantizar propuestas educativas para todos, con comunicaciones multidireccionales, síncronas y asíncronas. 


\section{Figura 1}

La población desconectada

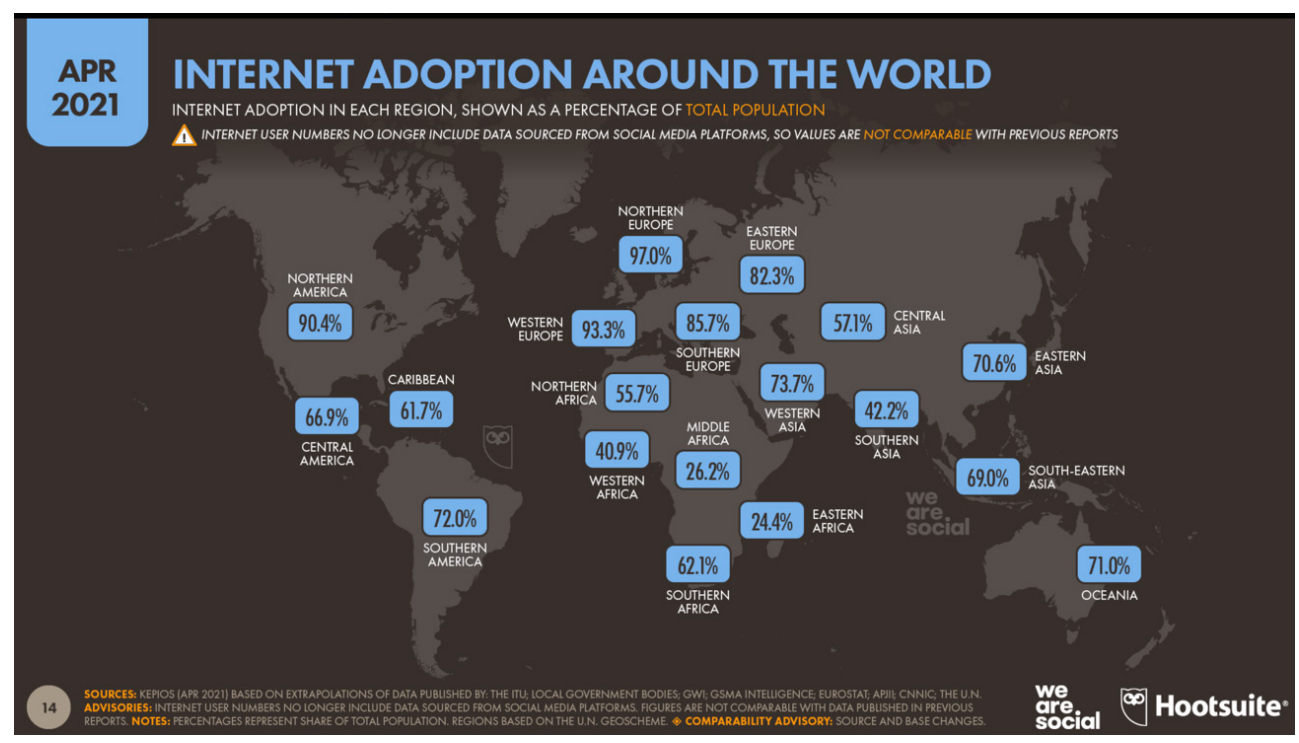

La realidad que muestra este informe es que más de 9 de cada 10 personas en el norte y oeste de Europa y América del Norte usan Internet en la actualidad, pero también muestra que más de 3 de cada 4 personas en África oriental permanecen desconectadas o, por ejemplo, más de mil millones de personas permanecen desconectadas en solo tres países del sur de Asia: India, Bangladesh y Pakistán.

En el Digital 2021 de Datareportal puede leerse que en enero de este año 2021 existía una población mundial de 7.830 millones de personas y 4.660 de ellas utilizan Internet (Kemp, 2021). La lectura negativa es que 3.270 millones no cuentan con esta ventaja.

Por otra parte, para cerciorarnos de la relevancia de estos medios más convencionales, UNICEF señala que, durante los diferentes períodos de confinamiento, la televisión fue utilizada por el $75 \%$ de los países y la radio fue una herramienta puesta en juego por el $58 \%$ de los países encuestados (Dreesen et al., 2020). En la Figura 2 se muestran los diferentes canales utilizados para el acceso al aprendizaje remoto. 


\section{Figura 2}

Canales múltiples para el acceso al aprendizaje remoto (2021)

\section{Percentage of countries using remote learning delivery channels (127 reporting countries)}

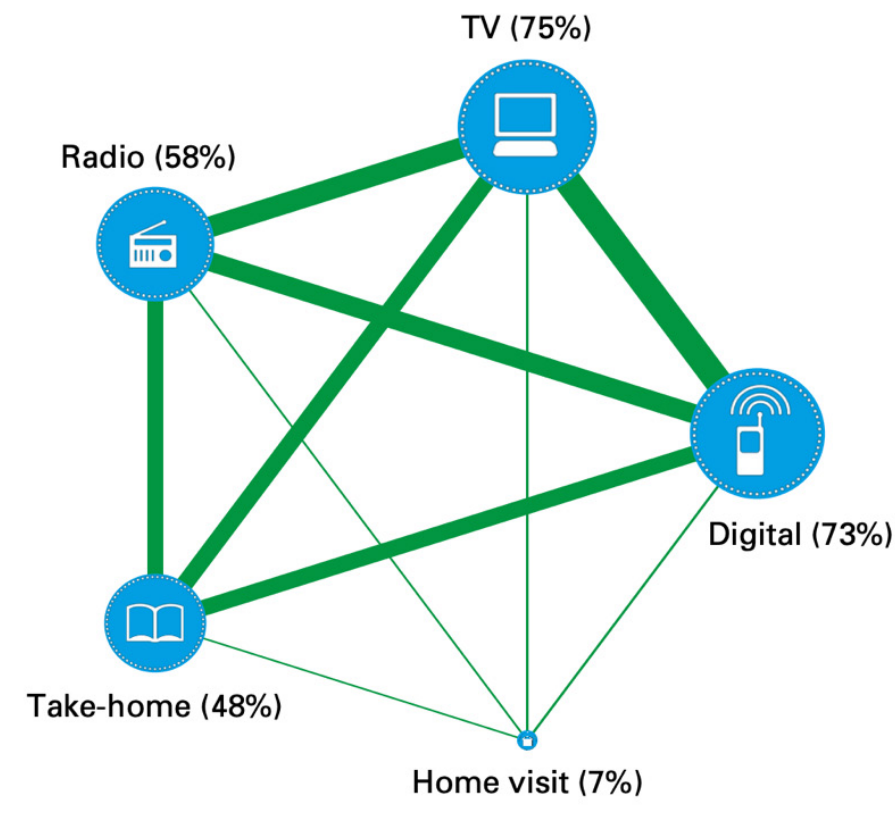

Source: UNICEF COVID-19 Education Response Survey - 127 countries.

Por su parte, un ilustrativo estudio del Banco Mundial (The World Bank, 2020) pone de relieve la importancia que la radio, la televisión, el audio y el vídeo tuvieron durante los tiempos más duros de la pandemia en un gran número de países. Programas de televisión y de radio, apoyados en la mayoría de las ocasiones por textos-guía o materiales impresos complementarios, y grabaciones tanto de radio como de televisión, que se hacían llegar a los estudiantes, fueron fórmulas eficaces ante la imposibilidad de mantener los habituales servicios de una educación presencial.

Más allá de que la sociedad y nuestro propio entorno educativo se sumergen hoy en lo digital, aquellas tecnologías de primera generación, analógicas, también cumplieron y pueden continuar respondiendo a determinadas necesidades 
educacionales. De ahí que, desde aquí, se postule el no dar por amortizados determinados recursos que hoy aún pueden cumplir su misión en determinados contextos del planeta. Desde este trabajo, se apuesta por integrarlos en propuestas educativas omnicanal, o multicanal, donde cada tecnología pueda dar respuesta a determinados contenidos, metodologías, necesidades sociales, etc. La radio y la televisión continúan siendo canales óptimos que complementan otras tecnologías digitales en los diferentes procesos de aprendizaje y en los que la motivación puede aumentar, dado que los estudiantes parecen estar más interesados y felices en atender el proceso de aprendizaje (Sarwinda et al., 2020).

\section{RADIO Y AUDIO}

Por encima del más escolar y académico de los recursos, por su larga historia (excepción hecha de la voz en directo del docente), que es el texto escrito, el documento sonoro en soporte tecnológico ha venido ocupando un destacado lugar en los sistemas educativos no presenciales. El lenguaje oral y la audición suponen vías principales para la comunicación, siempre y en todas las latitudes (García Aretio, 1994 y 2001). Hay casos en que el audio es esencial para mediar cierto tipo de información (Durbridge, 1984).

Si se mira a los recursos sonoros que históricamente fueron más útiles en las propuestas de educación a distancia, habría de hacerse mención, aunque sea breve, a un soporte de comunicación síncrona, el teléfono, que vino sirviendo para transmitir información, resolver problemas de los estudiantes, generar ideas, hacer preguntas y recibir respuestas, intercambiar y debatir (Flink, 1975; Ahlm, 1972; Punwar, 1972; Short, 1971). Al igual que los demás, este medio de comunicación ha ido evolucionando. En tiempos pretéritos las llamadas suponían un alto coste para el estudiante. Hoy, además de la comunicación inalámbrica y móvil, esa dificultad se ha subsanado gracias a las posibilidades que nos ofrecen diferentes soluciones digitales de mensajería instantánea.

\section{La radio}

La radio es un medio tecnológico de comunicación social que, a través de las ondas electromagnéticas genera una señal auditiva dirigida a masas, que se recibe de forma instantánea y simultánea. Siempre tuvo la radio educativa el propósito de acompañar el aprendizaje de los estudiantes $\mathrm{y}$, de forma especial, facilitar recursos educativos a aquellos más alejados de las zonas urbanas y con dificultades de acceso al bien de la educación (Arteaga Romero et al., 2004; Grupo de Trabajo sobre Tecnología e Innovación en la Educación, 2020). Pero, también en los países desarrollados, gracias a la digitalización, la radio contribuye a la mejora de los aprendizajes de aquellos estudiantes que, en cualquier lugar y en cualquier momento, pueden acceder a valiosos contenidos formativos a través del sonido y como fuente 
estimuladora de la imaginación, del fomento de la escucha y de la mejora de la expresión oral (Rodero-Antón, 2008).

Con su advenimiento en los inicios del siglo XX y su gran capacidad para llegar a todos, la radio se presentaba como un medio digno de tenerse en cuenta para los fines de la enseñanza a distancia (Grundin, 1984). Y esto, incluso, en la era de Internet, al mostrarse en muchos países y regiones del globo como un recurso muy interesante que ha sabido evolucionar y adaptarse a esta sociedad digital. Es éste un medio tecnológico que se ha venido utilizado por la mayoría de las universidades a distancia en el mundo y en otras muchas propuestas formativas de carácter no universitario, siguiendo en importancia al material impreso. Al tratarse de un recurso económico, versátil, de cobertura universal y de fácil disponibilidad, tuvo buen éxito en experiencias educativas no presenciales en diferentes países.

Ya en las primeras experiencias de las universidades a distancia, Jaminson y McAnany (1978) apuntaban tres destacadas ventajas de esos programas radiofónicos: mejoraban la calidad y relevancia de la educación, reducían los costes y afianzaban la democratización del acceso. También Tripp y Roby (1996) y Couch (1997) señalaban los beneficios de llegar a poblaciones dispersas, masivas y diversas y una clara economía de costes en aquellos procesos educativos. La ubicuidad es otra de las características fundamentales de la radio. El material impreso no puede manejarse en determinados entornos, en movimiento, desplazamientos, en el trabajo... No precisa el manejo de las manos (libro), ni de los ojos (vídeo, texto digital, pantalla...). Y el punto de la recreación del mensaje que se escucha, puede servir para otro debate (García Aretio, 2014).

Pero, como ya se señaló más arriba, la falta de interacción real de aquella radio educativa era una traba importante. No podía escucharse fuera de horario, no se podía pausar, volver atrás, preguntar, etc. (Chandar y Sharma, 2003; Damani y Mitchell, 2020). Otra dificultad estribaba en la posibilidad real de escuchar su programación, fuera porque no se sintonizaba la emisora o porque los horarios no eran compatibles con los del estudiante que trabajaba. Si ahora se dispone de conexión a Internet, la radio digital anula ese problema.

También hoy las emisiones radiofónicas de carácter educativo suelen convertirse en podcast de audio digitalizado que se soporta sin ningún problema en Internet. La radio vino a complementar el material impreso de las clásicas universidades a distancia, ofreciendo una información actualizada, dando la oportunidad de ofrecer sonidos originales de discursos, conferencias, entrevistas, debates..., y ha sido fundamental para conservar la voz de personajes ilustres (García Aretio, 1994 y 2001).

En tiempos de pandemia se aprovechó ese recurso con el fin de llegar a todos los rincones del globo (Núñez, 2021). Desde las Naciones Unidas (ONU, 2021) se asegura que, durante la pandemia de COVID-19, la radio permitió asegurar la continuidad de la educación y luchar contra la desinformación y, con seguridad, evitó que, durante la pandemia, miles de adolescentes y jóvenes abandonaran la escuela 
(Makazaga, 2020). Nadie podrá ocultar que la radio ha sido un medio educativo ideal en época del confinamiento. Hoy día, en muchas zonas de la geografía mundial, puede seguir siendo la radio un vehículo excepcional de comunicación, porque un pequeño transistor con unas pilas resuelve el problema, dado que no se hace precisa la electricidad ni, mucho menos, la conectividad a la red.

Algunas de las ventajas de la radio apuntadas hasta aquí, pueden ser consideradas sólo en aquellos contextos en los que la dificultad de contar con dispositivos digitales o con la conectividad necesaria, se hace patente. Pero otras abundan en beneficios que hoy día se mantienen como plausibles. En la Tabla 1 se ofrece un resumen de esas funciones, ventajas y posibilidades de la radio, ya expuestas, más algunas otras aportaciones, de Durbridge (1982), Cabero y Gisbert (2005), García Aretio (2001 y 2014), Bates, 2015; Makazaga (2020) y Damani y Mitchell (2020).

\section{Tabla 1}

Funciones y posibilidades de la radio educativa

\begin{tabular}{|c|c|}
\hline Penetración & $\begin{array}{l}\text { Facilidad para llegar a los más recónditos lugares. Masivo y de bajo } \\
\text { coste. }\end{array}$ \\
\hline $\begin{array}{l}\text { Información } \\
\text { actualizada }\end{array}$ & $\begin{array}{l}\text { Para ofrecer lo último y novedoso: bibliografía reciente, congresos, } \\
\text { simposios, legislación, acontecimientos políticos, sociales, culturales, } \\
\text { descubrimientos científicos o invenciones técnicas, nuevas corrientes y } \\
\text { teorías. }\end{array}$ \\
\hline Profundización & $\begin{array}{l}\text { En algún tema o aspecto que se acerque al típico seminario, incluso con } \\
\text { las aportaciones de especialistas variados }\end{array}$ \\
\hline $\begin{array}{l}\text { Presentación de } \\
\text { casos y ejemplos }\end{array}$ & $\begin{array}{l}\text { En los que el mensaje sonoro es bien adecuado y que por su actualidad } \\
\text { y significación merecen un comentario y discusión. }\end{array}$ \\
\hline $\begin{array}{l}\text { Personalidades de } \\
\text { relieve }\end{array}$ & $\begin{array}{l}\text { De relieve académico o histórico, cuya voz y estilo resultan } \\
\text { estimulantes para el alumno, quien tiene una sensación de } \\
\text { acercamiento e identificación. }\end{array}$ \\
\hline $\begin{array}{l}\text { Eficiencia. Coste/ } \\
\text { beneficio }\end{array}$ & $\begin{array}{l}\text { Eficiencia y rentabilidad por su positiva proporción coste- } \\
\text { beneficios. Especialmente en comunidades con escasas conectividad, } \\
\text { alfabetización digital o electricidad. }\end{array}$ \\
\hline $\begin{array}{l}\text { Debates, } \\
\text { coloquios }\end{array}$ & $\begin{array}{l}\text { Las entrevistas y los debates o coloquios suelen ser de un valor } \\
\text { singular, así como la presentación de variadas opiniones convergentes } \\
\text { o divergentes. }\end{array}$ \\
\hline Nuevas síntesis & $\begin{array}{l}\text { Aspectos integradores o concepciones no formuladas en otros } \\
\text { materiales, teniendo en cuenta las aportaciones recientes. }\end{array}$ \\
\hline Inmediatez & En la comunicación de noticias, avisos, informaciones. \\
\hline Ubicuidad & La portabilidad permite la escucha en lugares diferentes. \\
\hline $\begin{array}{l}\text { Fuerza } \\
\text { motivacional }\end{array}$ & $\begin{array}{l}\text { Aprovechamiento de la fuerza motivacional de la palabra y su } \\
\text { entonación. }\end{array}$ \\
\hline
\end{tabular}


Lección magistral Lección magistral, completa o complementaria a otros materiales. O bien, secciones de una determinada lección.

Otras ventajas Inmediatez en el mensaje, asequibilidad, versatilidad e inclusión.

\section{El audiocasete}

El audiocasete, hoy en desuso, desde inicio de los pasados años 6o, vino a complementar algunas limitaciones de la radio. Se trataba de una cinta encerrada en carcasa de plástico, de forma y tamaño estandarizado que permitía grabar y reproducir sonido, insertándola en un aparato que llevaba el mismo nombre. Su éxito fue incontestable, debido a su precio, solidez y fácil manejo (Race, 1998). Decía Bates (1984) que el recurso con mayor desarrollo en los doce últimos años en la Open University británica (desde su creación hasta la fecha de la publicación), había sido el humilde audiocasete.

Algunas de sus ventajas que se destacaban entonces, pueden ser aplicadas tanto a la radio como a los dispositivos y software que le sucedieron: walkman de Sony, cinta de audio digital (DAT), casete compacto digital (DCC), cartuchos de ocho pistas, $M P_{3}, C D-R W, W M A, W A V, A I F F$...

Universidades y centros de educación a distancia hicieron buen uso de este recurso, allá en las décadas de los años 70 y 80 del pasado siglo. En la Tabla 2 se muestran algunos valores de este recurso, por encima de los de la radio (Meacham y Butler, 1984; Laaser, 1986; Lappia, 1989; Kirschner et al., 1991; Lockwood, 1991; Kuomi, 2006; García Aretio, 1994 y 2001; Bates, 2015). Se muestra esta tabla, dado que la mayoría de estas posibilidades serán también propias del podcast.

\section{Tabla 2}

El audiocasete mostró ciertas ventajas

\begin{tabular}{ll}
\hline Fronteras & $\begin{array}{l}\text { Superaba las fronteras del tiempo y del espacio. Se podía usar cuándo } \\
\text { y dónde apeteciese. }\end{array}$ \\
\hline Transportabilidad & Fácilmente transportable. En un bolsillo cabían varias cintas. \\
$\begin{array}{l}\text { Control/ } \\
\text { Reversibilidad }\end{array}$ & $\begin{array}{l}\text { Su control era absoluto. Se podían escuchar los mensajes o partes } \\
\text { de ellos cuantas veces se desease o necesitase. Pausar y reproducir, } \\
\text { avanzar y retroceder según circunstancias del estudiante. }\end{array}$ \\
\hline $\begin{array}{l}\text { Flexibilidad/ } \\
\text { Perdurabilidad }\end{array}$ & $\begin{array}{l}\text { De tiempos y horarios. En cualquier lugar a cualquier hora. El mensaje } \\
\text { permanece, no desaparece. }\end{array}$ \\
\hline Objetivos & Podía cubrir diversidad de objetivos y contenidos de aprendizaje. \\
\hline Apoyo & A otros medios, fundamentalmente al material impreso. \\
\hline $\begin{array}{l}\text { Grabación/ } \\
\text { Reproducción }\end{array}$ & $\begin{array}{l}\text { Muchos de sus contenidos eran de facilísima producción. Bastaba con } \\
\text { colocar el dispositivo y grabar lo que acontecía: conferencia, discurso, } \\
\text { acontecimientos, etc. }\end{array}$ \\
\hline
\end{tabular}




\begin{tabular}{ll}
\hline Modificación & Posibilidad de modificar el mensaje, borrando, grabando, ampliando... \\
\hline $\begin{array}{l}\text { Tutoría verbal - } \\
\text { Bidireccionalidad }\end{array}$ & $\begin{array}{l}\text { El correo postal permitía intercambiar casetes con respuestas, } \\
\text { trabajos, actividades. Se llegó a utilizar como material de tutoría } \\
\text { verbal sonora. }\end{array}$ \\
\hline Facilidad manejo & Su uso no precisaba de conocimientos técnicos especiales. \\
\hline
\end{tabular}

\section{El Podcast}

Se trajo a este trabajo el audiocasete, ya obsoleto, porque, de alguna manera, fue precursor del podcast. En cuanto a este recurso, recuérdese que este término, podcast, fue declarado como la «Palabra del Año 2005" por los editores del New Oxford American Dictionary según la cadena BBC de noticias, dada la facilidad que tiene cualquier persona que cuente con un dispositivo de grabación y una conexión a Internet, para producir un podcast. Esa conexión a Internet y el imparable aumento de los dispositivos móviles, han impulsado la creciente penetración del fenómeno podcast (podcasting) que, desde la perspectiva educativa, debe aprovecharse como recurso idóneo para el aprendizaje también, desde propuestas de mobile learning (Piñeiro-Otero, 2012). Esta autora repasa literatura científica abundante que muestra la efectividad del recurso, especialmente en ambientes universitarios. El enriquecimiento de la experiencia de aprendizaje parece probado (Boulos et al., 2006; Salmon y Edirisingha, 2008).

A nivel internacional se viene utilizando en bastantes universidades dadas sus ventajas, porque integra la mayoría de las funciones y posibilidades señaladas para la radio y para el audiocasete, entre las que, en este caso, podrían destacarse (García Aretio, 2001 y 2014; Borges, 2009):

- Mejora de la comunicación, de la relación personal y el aprendizaje colaborativo.

- Aumento de la motivación y fomento del aprendizaje autónomo y significativo.

- Ayuda a la autogestión y organización del tiempo.

- Resulta sencillo grabar y reproducir cualquier tipo de contenido sonoro, con posibilidades de escucha reiterada, pausa, vuelta atrás...

Puede concluirse este apartado señalando que las características pedagógicas del audio se han mantenido constantes durante un período bastante largo. Sin embargo, parece que en la actualidad no se viene explotando suficientemente el audio como medio educativo. Quizás el COVID-19 pueda haber sido un revulsivo, pretexto u oportunidad para la potenciación de estos medios como recursos educativos relevantes. 


\section{TELEVISIÓN Y VÍDEO}

La imagen, sea dibujada en el encerado, proyectada o en papel, siempre tuvo relevancia en la educación, generalmente acompañada por el texto o por la voz del profesor. La radio o audio, apoyados en determinados textos (o textos apoyados en esos programas radiofónicos o audios), aumentan el poder de la comunicación educativa o educomunicación.

Si se sube un escalón más, las imágenes en movimiento del cinematógrafo, posteriormente acompañadas de sonido, supusieron un recurso didáctico incuestionable para aquellas poblaciones o grupos que pudieron disponer del mismo. Pero el cine, las películas, cortos, documentales, etc., tenían un escaso recorrido por la dificultad de generalizar su uso. Por eso, se salta en este apartado a recursos de uso más generalizado en la sociedad: la televisión y el vídeo.

\section{La televisión}

La televisión, para poblaciones sin acceso a Internet, o para quienes siguen aquellos programas educativos que la vienen utilizando como complemento o recurso educativo, se configura como un medio de relevancia educativa indiscutible dado su alcance masivo y posibilidades didácticas. Y, especialmente, en la educación a distancia, por sus características de amplia cobertura de audiencia, brindando igualdad de oportunidades y transmitiendo a una gran masa de audiencia (Saglik y Ozturk, 2001). Por otra parte, la presentación de los contenidos procedimentales resulta más eficaz si se hace a través de los medios audiovisuales que sólo con texto (Carmichael et al., 2018).

Además, igual que sucedió con la radio, programas de televisión que pueden ser utilizados en contextos educativos, hoy pueden integrarse en la red desde cualquier plataforma o canal institucional, y así el estudiante puede acceder al mismo en cualquier momento y lugar (Grupo de Trabajo sobre Tecnología e Innovación en la Educación, 2020).

Desde siempre se consideró que la televisión cuenta con un destacado papel en los entornos educativos no presenciales, debido a sus características distintivas de entrega, presentación y control (Bates, 1988). Watson y McIntyre (2020) nos acercan evidencias surgidas sobre la televisión educativa, tras una revisión generada con motivo del COVID-19. Se destacan las siguientes:

- Puede beneficiar a los resultados de aprendizaje de los estudiantes en las materias básicas.

- Mejorar la capacidad de razonamiento social.

- La co-visualización (ver la televisión con otros) podría proporcionar beneficios tanto directos como indirectos.

- En países con escasos recursos económicos, la evidencia sugiere que el acceso a contenidos de televisión educativa es relativamente alto. 
En un interesante artículo en el New York Times, Mueller y Taj (2020) apuntan que, desde que comenzó la pandemia, muchos países recurrieron a la educación por televisión con muy diversas estrategias: desde combinaciones de clases en línea y televisadas (China) hasta grabaciones de clases desde el aula, programas específicos, animaciones (Tanzania), etc.; desde propuestas locales hasta decisiones a nivel país; desde programas enfocados a un solo grupo de edad hasta planes de estudio para todos los grados a través de la televisión (Perú). En este último país, donde solo el 15 por ciento de los estudiantes de escuelas públicas tiene acceso a una computadora en casa, las clases transmitidas por televisión se convirtieron en el modo de aprendizaje dominante durante la pandemia. Incluso en Estados Unidos (New Jersey) se produjeron programas especiales de TV tras conocerse que 300.000 niños no contaban con acceso a Internet.

En países con pobre cobertura de Internet (en Indonesia un tercio de la población no tiene acceso) se emitieron programas educativos desde la televisión para escolares de todas las edades. Por su parte, el Centro de Medios Educativos de Amazonas, en Brasil, informó que 4,5 millones de niños de la zona siguieron sus lecciones televisadas durante la pandemia.

Aunque no cabe duda de que, a los efectos de educación y formación, la televisión de antes contaba con algunos inconvenientes (García Aretio, 2001):

- Colocaba al televidente en situación muy pasiva al no permitir el feedback. Era un medio efímero, con carácter no repetible, ni revisable.

- Sometía al estudiante a unos horarios fijos de emisión. Era un medio rígido, lineal y progresivo que no permitía pausas.

- Era un medio continuo que obligaba a pensar a una velocidad predeterminada.

- Las emisiones de cada curso o programa eran escasas, porque generalmente iban dirigidas a grandes grupos muy heterogéneos.

- Los costes de producción y transmisión eran muy elevados.

\section{El vídeo}

Para subsanar esos problemas señalados, nació el vídeo, muy ligado con la televisión, bien fuese para grabar, almacenar o reproducir emisiones de televisión, bien por necesitar del receptor de televisión para su difusión (Cebrián, 1994). Integrar oído y vista, por una parte, y el texto, audio e imágenes por otra, en un mismo recurso educativo, puede imaginarse que potenciará la capacidad de aprender.

El vídeo inicialmente se presentaba en sus diferentes formatos analógicos: VHS, U-MATIC, BETACAM, Super-8... Los posteriores sistemas digitales posibilitaron el almacenamiento y reproducción desde diferentes soportes que, además de su calidad muy superior, no se deterioraban por uso-reproducción o por el paso del tiempo, como el $D V D, B l u-R a y . .$. Y lo que ya es más habitual, vídeo soportado en la red, que puede propiciar estrategias didácticas específicas de utilización, ya sea desde la 
reproducción de determinados programas de televisión, ya sea desde la utilización de vídeos didácticos (Ferrés, 1994), que hoy tanto abundan en la red y que pueden ser de producción propia o ajena.

No podría ocultarse el crecimiento exponencial de este recurso en las diferentes propuestas formativas, no sólo a distancia, aunque en esta modalidad se han incrementado de forma extraordinaria. Basta con acercarse a los repositorios de vídeos de universidades y otras instituciones o a la cantidad de programas educativos existentes, formales o reglados. Pero también, y sobre todo, programas, tutoriales y cursos de carácter no formal, de perfeccionamiento, actualización, reciclaje, etc. Véanse, por ejemplo, los MOOC. El recurso vídeo ha ido a más y seguirá creciendo en posibilidades y eficacia, tanto en educación a distancia, como en la presencial o en la híbrida (blended).

Respecto a los formatos en que se suelen presentar los vídeos educativos, varían. (Guo et al., 2014, Chorianopoulos, 2018):

- simple grabación directa de la docencia en el aula, desde la pizarra o pizarrón, con una cámara fija;

- busto parlante desde el escritorio o mesa;

- mediante screencasts (grabación digital de la pantalla del ordenador) con voz en off;

- presentación de diapositivas, con o sin audio;

- postproducción de vídeos altamente elaborada con imágenes o técnicas de combinación de vídeos.

También deben considerarse los diferentes tipos de presencia humana en el vídeo, docente o expositor a cuerpo entero, busto, cabeza en una esquina de la pantalla, avatar, robot parlante, etc. De un potente estudio sobre la plataforma edX $M O O C$ se muestran algunas conclusiones de interés (Guo et al., 2014):

- Los vídeos más cortos son mucho más atractivos que los largos,

- Los vídeos que intercalan la cabeza o imagen del profesor con diapositivas son mejores que aquellos que sólo muestran diapositivas.

- Los vídeos con un toque personal pueden ser más interesantes para el estudiante que aquellos producidos en estudio.

- Los vídeos, que muestran a los instructores hablando con bastante rapidez y entusiasmo, atraen más.

El vídeo educativo es, cada vez más, una destacada herramienta en el diseño pedagógico, pero generalmente debe ser complementado con otros recursos. Por ello, a la hora de seleccionar el vídeo concreto, debe tenerse una visión completa que considere el tipo e índole del curso y las necesidades de los estudiantes (Chorianopoulos, 2018). 
En fin, las posibilidades formativas de vídeo y televisión son indudables. Como síntesis de lo expuesto, y enriquecido con otras aportaciones (McKenzie et al., 1979; Hizal, 1983; Bates, 1988; Cabero, 1994; Race, 1994 y 1998; García Aretio, 2001; Saglik y Ozturk, 2001; Koumi, 2006; Schwartz y Hartmann, 2007; Bates, 2015), se proponen algunas de las funciones que hoy pueden aprovecharse de estos medios (Tabla 3).

\section{Tabla 3}

\section{Funciones y posibilidades de televisión y vídeo}

\begin{tabular}{|c|c|}
\hline Introducción & Introducir temas, apoyar documentos o textos, sugerir actividades. \\
\hline Integración & Integrar recursos como el texto, la imagen fija y animada, y el audio. \\
\hline Complemento & Complementar o ampliar temas de estudio. \\
\hline Igualdad & $\begin{array}{l}\text { Fomentan el principio de igualdad de oportunidades ante el mensaje } \\
\text { educativo. }\end{array}$ \\
\hline Competencias & $\begin{array}{l}\text { Propician el logro de competencias comunicativas, de representación, } \\
\text { procedimentales... }\end{array}$ \\
\hline Realidad & $\begin{array}{l}\text { Presentan y pueden ayudar a reorganizar la propia realidad y mostrar } \\
\text { a personalidades, actitudes, lenguaje corporal, situaciones reales de la } \\
\text { vida, etc. }\end{array}$ \\
\hline Motivación & Suponen un elemento motivador de primer orden. \\
\hline Ver al docente & $\begin{array}{l}\text { Llevan la imagen del profesor o experto allá donde sería difícil contar } \\
\text { con ese tipo de mensaje. }\end{array}$ \\
\hline Demostraciones & $\begin{array}{l}\text { Permiten presenciar la demostración de experiencias o de situaciones } \\
\text { experimentales, procesos, relaciones causa-efecto, }\end{array}$ \\
\hline Debates & $\begin{array}{l}\text { Presentan casos o argumentos que puedan apoyar y reforzar los } \\
\text { aprendizajes o sean motivo de debate. Otras visiones o corrientes. }\end{array}$ \\
\hline $\begin{array}{l}\text { Fuentes } \\
\text { primarias }\end{array}$ & Posibilitan disfrutar de documentos primarios. \\
\hline Movimiento & $\begin{array}{l}\text { Facilitan apreciar los principios que implican una dinámica cambiante } 0 \\
\text { en movimiento. }\end{array}$ \\
\hline $\begin{array}{l}\text { Ayuda a lo } \\
\text { abstracto }\end{array}$ & Aclaran principios abstractos utilizando modelos físicos. \\
\hline Dimensionalidad & $\begin{array}{l}\text { Permiten observar principios que impliquen espacios de dos o más } \\
\text { dimensiones. }\end{array}$ \\
\hline $\begin{array}{l}\text { Aceleración/ } \\
\text { Ralentización/ } \\
\text { Pausa }\end{array}$ & $\begin{array}{l}\text { Admiten reproducir al ralentí o acelerado para demostrar los cambios } \\
\text { en el curso del tiempo. Así como pausar y repetir cuantas veces sea } \\
\text { preciso. }\end{array}$ \\
\hline Condensación & Sintetizan en un todo coherente una gran gama de informaciones. \\
\hline Atención a masas & Puede llegar a grandes masas. \\
\hline
\end{tabular}


En los últimos años la llegada de los MOOC, la proliferación de dispositivos móviles, las plataformas de vídeo y la expansión de la banda ancha, han impulsado el uso del vídeo, además de que tanto docentes como estudiantes se han convertido en productores y consumidores de vídeo (Villa et al., 2020). Y desde 2020, tras la crisis sanitaria vivida en el globo, estos recursos han tomado mayor relevancia educativa.

En todo caso, si se buscan finalidades educativas, resulta crucial, a la hora de primar el vídeo en nuestra acción pedagógica, el integrarlo plenamente en la planificación y diseño del programa del curso o asignatura. A pesar de todas las posibilidades y ventajas señaladas, se coincide con la opinión de Laaser y Toloza (2017) respecto a que el potencial del vídeo no se ha explotado, ni mucho menos, lo suficiente en entornos educativos.

Sin embargo, por ejemplo, sí que fue y es bien explotado el recurso por parte del proyecto Khan Academy. Salman Khan quizás pensó que la simulación de una simple pizarra negra bien utilizada y sirviéndonos de las ventajas de Internet, podría dar buenos resultados para el aprendizaje, inicialmente, de las matemáticas. Ese afán se convirtió posteriormente en la Khan Academy (KA), que se configuró como un gran canal de recursos educativos abiertos, con un gran potencial de llegar a decenas de miles de estudiantes de todo el mundo. Ya en 2015 llegaba a más de un millón de profesores y diez millones de estudiantes en todo el mundo. Los recursos de esta experiencia audiovisual están mostrándose traducidos a más de 30 idiomas diferentes. En un reciente estudio, se concluye que KA es eficaz para promover la personalización, la independencia y los procesos innovadores de enseñanzaaprendizaje (Vidergor y Ben-Amram, 2020). En todo caso, resulta ser un recurso complementario de la enseñanza en el aula o, fundamental, cuando se carece de la posibilidad de acudir a centros educativos.

\section{La videoconferencia}

Llegados a este punto, y sin profundizar en ello, cabría mencionar, dentro del grupo de recursos audiovisuales, uno de ellos que ha explosionado en estos últimos meses: el de las videoconferencias, teleconferencias, videollamadas, webinars, etc. La videoconferencia como instrumento de aprendizaje se viene utilizando con el fin de facilitar comunicaciones eficaces entre estudiantes y docentes, o de estudiantes entre sí, especialmente cuando la relación presencial no era posible (Al-Samarraie, 2019).

Y han sido indudables las interacciones, especialmente síncronas, que se vienen realizando y potenciando en estos últimos meses a raíz del confinamiento, las videollamadas a través de las diferentes soluciones informáticas que se han relanzado. Si bien la educación a distancia las vino utilizando con anterioridad a la pandemia, es ahora cuando casi todos los sectores y niveles educativos han hecho uso de ellas. En estos formatos educativos mediados, las conexiones por videoconferencia supusieron un gran aporte al acercar de forma evidente la "presencia" del profesor 
con su mensaje audiovisual a ese estudiante alejado en el espacio y, probablemente, en el tiempo (Romero, 2018; Chorianopoulos, 2018) que aminoraba el recurrente sentimiento de soledad en estos sistemas a distancia (Chen et al., 2020) y que, además, parece que los estudiantes aceptan de buen grado (Fatani, 2020). El confinamiento exigió del profesorado la adopción de otras estrategias didácticas a través de recursos diferentes. La videoconferencia síncrona parecía uno de los más adecuados, al menos porque podía asemejarse más a la clase presencial (Sandars et al., 2020).

Como podrá valorarse, este recurso integra todas las ventajas señaladas para la televisión y el vídeo, además porque puede hacerlo, permitiendo una interacción más auténtica (Reaburn y McDonald, 2017), agregándose la de la comunicación bidireccional, multidireccional, y en sincronía (Reese y Chapman, 2017). Sin embargo, haría falta capacitación para saber seleccionar los mejores sistemas de videoconferencia en educación, así como para explorar posibilidades y estrategias didácticas aplicables en formatos educativos no presenciales (Correia et al., 2020).

\section{CONCLUSIÓN}

La educación a distancia a nivel mundial fue pionera en el uso educativo de los recursos de radio y televisión, audio y vídeo. Sus prestaciones educativas resultan relevantes y, en ocasiones, imprescindibles para la aprehensión de determinados aprendizajes y adquisición de las competencias concomitantes. La radio y el audio son recursos asequibles, de reducido coste, de fácil generación y recepción e insustituibles para determinadas poblaciones alejadas de conectividad y, quizás, de energía eléctrica. También útiles en contextos educativos sin esas privaciones.

Un peldaño superior en costes, dificultades de creación, emisión y recepción tienen la televisión y el vídeo. Pero son recursos de uso masivo en la práctica totalidad de hogares. El valor de la imagen, del movimiento, de la replicabilidad de procesos o realidades, etc., resultan un instrumento de gran valor educativo.

El COVID-19 y los subsiguientes confinamientos y restricciones de movilidad ha supuesto un incuestionable impulso a estos recursos educativos que salvan distancias, acercan la interacción y aprovechan las inmensas posibilidades del audio, del vídeo, de la sincronía y asincronía, para dar respuestas a propuestas educativas de la más diversa índole. Hoy, los sistemas digitales pueden alojar, grabar y reproducir cualquier tipo de documento sonoro, y esto último, sea en formato síncrono (en directo) o asíncrono (en diferido).

Pero no se olvide que toda innovación cuenta con sus dificultades. Por ejemplo, si de producir vídeos se tratase, habría de contemplarse un inconveniente importante: el de la dedicación que se precisa en su diseño, desarrollo, producción, etc. Aunque sería un trabajo que podrá tener efecto multiplicador, dado el número de ocasiones en que puede utilizarse con finalidades educativas. Menor esfuerzo supondría la 
búsqueda, selección, encaje en la programación etc., de materiales de libre acceso a través de Internet.

Porque hoy son evidentes las posibilidades que están abriendo las operadoras de televisión digital a través de Internet, sea en vivo o en diferido, más allá de la inmensa videoteca que nos ofrece la red. Sin necesidad de producir, en la red puede encontrarse casi todo aquello que se precisa y de forma gratuita, en gran medida, tanto en audio como en vídeo. Puede entenderse que esa magnitud de recursos no se ha llegado a explotar de forma sistemática y programada en la generalidad de instituciones educativas.

\section{REFERENCIAS}

Ahlm, M. (1972). Telephone instruction in correspondence. Epistolodidaktika, 2, 49-64.

Al-Samarraie, H. (2019). A Scoping Review of Videoconferencing Systems in Higher Education: Learning Paradigms, Opportunities, and Challenges. International Review of Research in Open and Distributed Learning, 2O(3). https:// doi.org/10.19173/irrodl.v20i4.4037

Arteaga Romero, C., Martínez Hernández, C. A., Medel Hernández, N. R., Piña Camacho, H. I., y Soto Godínez, B. (2004). La radio como medio para la educación. Razón y Palabra, 36.

Bartolomé, A. (2000). Informar y comunicar en los procesos comunicativos del siglo XXI. Actas XII Congreso Nacional y I Iberoamericano de Pedagogía (Tomo II). Sociedad Española de Pedagogía.

Bates, A. W. (1984). Broadcasting in Education: An Evaluation. Constable.

Bates, A. W. (1988). Television, Learning and Distance Education. Journal of Educational Television, 14(3), 213-225. https://doi. org/10.1080/0260741880140305

Bates, A. W. (2015). Teaching in a Digital Age. BCampus.

Baum, S., y McPherson, M. (2019). The Human Factor: The Promise \& Limits of Online Education. Daedalus, 148(4),
235-254. https://doi.org/10.1162/ daed a 01769

Borges, F. (2009). Profcast: Aprender y enseñar con podcast. UOC.

Boulos, M., Maramba, I., y Wheeler, S. (2006). Wikis, blogs and podcasts: a new generation of Webbased tools for virtual collaborative clinical practice and education. BMC Medical Education, 41(6). https://doi.org/10.1186/14726920-6-41

Cabero, J. (1994). Retomando un medio: la televisión educativa. En CMIDE-SAV. Medios de comunicación, recursos y materiales para la mejora educativa. Sevilla: CMIDE y SAV.

Cabero, J., y Gisbert, M. (2005). Materiales formativos multimedia en la red. Guía práctica para su diseño. Eduforma/ Trillas.

Carmichael, M., Reid,A.,y Karpicke,J.(2018). Assessing the impact of educational video on student engagement, critical thinking and learning: The current state of play. SAGE Publishing. https://bit.ly/39q3Lt5

Cebrián, M. (1994). Los vídeos didácticos: claves para su producción y evaluación. Pixel-Bit. Revista de Medios y Educación, 1, 31-42.

Chandar, U., y Sharma, R. (2003). Bridges to Effective Learning Through Radio. The International Review of Research 
in Open and Distributed Learning, 4(1). https://doi.org/10.19173/irrodl.v4i1.118

Chen, J., Dobinson, T., y Kent, S. (2020). Student's perspectives on the impact of Blackboard Collaborate on Open University Australia (OUA) online learning. Journal of Educators Online, $\quad 17(1) . \quad \underline{\text { http://hdl.handle. }}$ net/20.500.11937/77825

Chorianopoulos, K. (2018). A Taxonomy of Asynchronous Instructional Video Styles. The International Review of Research in Open and Distributed Learning, 19(1). $\quad$ https://doi.org/10.19173/irrodl. v19i1.2920

Correia, A.P., Liu, C., y Xu, F. (2020). Evaluating videoconferencing systems for the quality of the educational experience. Distance Education, 41(4), 429-452. https://doi.org/10.1080/01587919.2020 .1821607

Couch, L. (1997). Digital and Analog Communication Systems. Upper Saddle River, NJ.: Prentice-Hall,

Damani, K., y Mitchell, J. (2020). Radio: Rapid Evidence Review. EdTechHub. https://edtechhub.org/rapid-evidencereview-radio/

Datareportal (2021). Digital 2021. April Global Statshot Report. https:// datareportal.com/reports/tag/ Digital+2021+April+Statshot

Dreesen, T., Akseer, S., Brossard, M., Dewan, P., Giraldo, J.P., Kamei, A., Mizunoya, S., y Ortiz, J. S. (2020). Promising practices for equitable remote learning Emerging lessons from COVID-19 education responses in 127 countries. Innocenti Research Brief. UNICEF.

Durbridge, N. (1982) Audio-cassettes in Higher Education. Milton Keynes: The Open University.

Durbridge, N. (1984) Audio-cassettes. En A. Bates (Ed.) The Role of Technology in Distance Education. Croom Hill/St Martin's Press.
Fatani, T. (2020). Student satisfaction with videoconferencing teaching quality during the COVID-19 pandemic. BMC Medical Education, 20. https://doi.org/10.1186/ s12909-020-02310-2

Ferrés, J. (1994). Televisión y educación. Paidós.

Flink, R. (1975). The Telephone as an Instructional Aid in Distance Education; A Survey of the Literature. Lund Univ.

García Aretio, L. (1994). Educación a distancia hoy. UNED.

García Aretio, L. (2001). Educación a distancia. De la teoría a la práctica. Ariel.

García Aretio, L. (2014). Bases, mediaciones y futuro de la educación a distancia de la sociedad digital. Síntesis. https://doi. org/10.5944/ried.2.1.2084

Grundin, H. (1984). Radio. En A. W. Bates, The rol of technology in distance education. Croom Helm.

Grupo de Trabajo sobre Tecnología e Innovación en la Educación (2020). Estrategia multicanal: El rol de la radio y la televisión en la educación a distancia. Diálogo Interamericano.

Guo, P. J., Kim, J., y Rubin, R. (2014, March). How video production affects student engagement: An empirical study of mooc videos. En Proceedings of the First ACM Conference on Learning@ Scale Conference, (pp. 41-50). ACM. https:// doi.org/10.1145/2556325.2566239

Hizal, A. (1983). Uzaktan Ogretim Surecleri ve Yazili Gerecler [Distance Teaching Process and Print Materials]. Ankara Universitesi Egitim Fakultesi Yayinlari.

Jaminson, D., y McAnany, E. (1978). Radio for Education and Development. Sage.

Kemp, S. (2021). Digital 2021: Global Overview Report. https://datareportal. com/reports/digital-2021-globaloverview-report

Kirschner, P., Brink, H. V., y Meester, M. (1991). Audiotape feedback for essays in distance education. Innovative Higher 
Education, 15, 185-195. https://doi. org/10.1007/BFoo898030

Koumi, J. (2006). Designing video and multimedia for open and flexible learning. Routledge. https://doi. org/10.4324/9780203966280

Lappia, A. (1989). Audio.cassette tapes in distancia teaching: student evaluation. Distance education, 1O(2), 277-284. https://doi. org/10.1080/0158791890100211

Laaser, W. (1986) Some didactic aspects of audio-cassettes in distance education. Distance Education, 7(1), 143-152. https:// doi.org/10.1080/0158791860070110

Laaser, W., y Toloza, E. A. (2017). The Changing Role of the Educational Video in Higher Distance Education. The International Review of Research in Open and Distributed Learning, 18(2). https:// doi.org/10.19173/irrodl.v18i2.3067

Lockwood, F. (1991). Data Collection in Distance Education Research: The Use of Self-Recorded Audiotape. Deakin Univ.

Makazaga, I. (2020): La radio, aliada contra la pandemia en África. Planeta Futuro. El País. http://bit.ly/3npz4cm

Meacham, E., y Butler, A. (1984). Audio Tapes for Distance Education. Wagga Wagga

McKenzie, N., Postgate, R., y Schuphan, J. (1979). Enseñanza abierta. Sistemas de enseñanza postsecundaria a distancia. UNESCO.

Mueller, B., y Taj, M. (2020). La educación por televisión vive un auge por la pandemia del coronavirus. The New York Times. https://www.nytimes.com/ es/2020/08/17/espanol/educaciontelevision.html

Núñez, F. (2021). Radio in Education in the Face of COVID-19. Observatory. TECMonterrey. https://observatory.tec.mx/ edu-bits-2/radio-education-and-covid19

ONU (2021). World Radio Day 13 February. https://www.un.org/en/observances/ radio-day
Piñeiro-Otero, T. (2012). Los podcast en la educación superior. Hacia un paradigma de formación intersticial. Revista Iberoamericana de Educación, 58(1), 1-12. https://doi.org/10.35362/ rie 5811462

Punwar, A. (1972). The telephone - radio conference: A method of continuing education for health professional. The American Journal of Occupational Therapy, 26(8), 410-414.

Race, P. (1994). The open learning handbook ( $2^{\text {nd }}$ edition). Kogan Page.

Race, P. (1998). Interesting ways to write open learning materials. Bristol: Technical and Educational Services Ltd.

Reaburn, P., y McDonald, J. (2017). Creating and facilitating communities of practice in higher education: Theory to practice in a regional Australian university. En J. McDonald, y A. Cater-Steel (Eds.), Communities of Practice. Springer, Singapore. https://doi.org/10.1007/978981-10-2879-3 6

Reese, R. J., y Chapman, N. (2017). Promoting and evaluating evidence-based telepsychology interventions: Lessons learned from the university of Kentucky telepsychology lab. En M. Maheu, K. Drude, y S. Wright (Eds.), Career paths in telemental health (255-261). Springer. https://doi.org/10.1007/978-3-31923736-7 26

Rodas, B. I., y Celleri, A. A. (2017). Influencia de la radio con un enfoque educomunicativo para la formación ciudadana. INNOVA Research Journal, 2(8.1), 223-235. https://doi. org/10.3389o/innova.v2.n8.1.2017.367

Rodero-Antón, E. (2008). Educar a través de la radio. Signo y Pensamiento, 27(52), 97-109.

Romero, J. (2018). Videollamada como recurso tecnológico para la educación superior a distancia. Red de Investigación Educativa, 11(1), 56-61. 
Saglik, A., y Ozturk, A. (2001). La televisión como tecnología educativa: uso de la televisión en la facultad de educación abierta, Universidad de Anadolu. Revista turca en línea de educación a distancia, 2(1).

Salmon, G., y Edirisingha, P. (2008). Podcasting for Learning in Universities. McGraw Hill Education.

Sandars, J., Correia, R., Dankbaar, M., de Jong, P., Goh, P. S., Hege, I., y Pusic, M. (2020). Twelve tips for rapidly migrating to online learning during the COVID-19 pandemic. Med Publish. https://doi. org/10.15694/mep.2020.000082.1

Sarwinda, K., Rohaeti, E., y Fatharani, M. (2020). The development of audiovisual media with contextual teaching learning approach to improve learning motivation and critical thinking skills. Psychology, Evaluation, and Technology in Educational Research, 2(2), 98-114. https://doi.org/10.33292/petier.v2i2.12

Schwartz, D. L., y Hartman, K. (2007). It's not Video Anymore: Designing Digital Video for Learning and Assessment. En R. Goldman, R. Pea, B. Barron, y S. J. Derry (Eds.), Video Research in the Learning Sciences (335-348). Erlbaum.

Short, J. (1971). Teaching by telephone: The problems of teaching without the visual channel. Teaching at a distance, 3 .
The World Bank (2020). How countries are using edtech (including online learning, radio, television, texting) to support access to remote learning during the COVID-19 pandemic. Education and Technology. The World Bank. https://bit.ly/2XJtXfl

Tripp, S., y Roby, W. (1996). Auditory presentations in language laboratories. En D. H. Jonassen (Ed.), Handbook of research for educational communications and technology (821-850). Simon y Schuster Macmillan.

UNESCO (2020). El aprendizaje por conducto de la radio y la televisión en tiempos del COVID-19. UNESCO. https://es.unesco.org/news/aprendizajeconducto-radio-y-television-tiempos-delcovid-19

Vidergor, H. E., y Ben-Amram, P. (2020). Khan Academy effectiveness: The case of math secondary students' perceptions. Computers \& Education, 157. https://doi. org/10.1016/j.compedu.2020.103985

Villa, M. I., Marulanda, A., y Molina, T. (2020). La experimentación educativa, social y técnica en los medialabs universitarios. Revista Complutense de Educación, 31(2), 231-240. https://doi. org/10.5209/rced.62114

Watson, J., y McIntyre, N. (2020). Educational Television: Rapid Evidence Review. EdTechHub. https://zenodo.org/ record/3956366\#.YQgO4VP7Tfg 